\title{
A Message Ordering Problem in Parallel Programs ${ }^{\star}$
}

\author{
Bora Uçar and Cevdet Aykanat \\ Department of Computer Engineering, Bilkent University, 06800, Ankara, Turkey \\ \{ubora, aykanat\}@cs. bilkent. edu.tr
}

\begin{abstract}
We consider a certain class of parallel program segments in which the order of messages sent affects the completion time. We give characterization of these parallel program segments and propose a solution to minimize the completion time. With a sample parallel program, we experimentally evaluate the effect of the solution on a PC cluster.
\end{abstract}

\section{Introduction}

We consider a certain class of parallel program segments with the following characteristics. First, there is a small-to-medium grain computation between two communication phases which are referred to as pre- and post-communication phases. Second, local computations cannot start before the pre-communication phase ends, and the post-communication phase cannot start before the computation ends. Third, the communication in both phases is irregular and sparse. That is, the communications are performed using point-to-point send and receive operations, where the sparsity refers to small number of messages having small sizes. These traits appear, for example, in the sparse-matrix vector multiply $y=A x$, where matrix $A$ is partitioned on the nonzero basis and also in the sparse matrix-chain-vector multiply $y=A B x$, where matrix $A$ is partitioned along columns and matrix $B$ is partitioned conformably along rows. In both examples, the $x$-vector entries are communicated just before the computation and the $y$-vector entries are communicated just after the computation.

There has been a vast amount of research in partitioning sparse matrices to effectively parallelize computations by achieving computational load balance and by minimizing the communication overhead [2-4, 7,8]. As noted in [7], most of the existing methods consider minimization of the total message volume. Depending on the machine architecture and problem characteristics, communication overhead due to message latency may be a bottleneck as well [5]. Furthermore, the maximum message volume and latency handled by a single processor may also have crucial impact on the parallel performance [10,11]. However, optimizing these metrics is not sufficient to minimize the total completion time of the subject class of parallel programs. Since the phases do not overlap, the receiving time of a processor, and hence the issuing time of the corresponding send operation play an important role in the total completion time.

\footnotetext{
* This work is partially supported by the Scientific and Technical Research Council of Turkey (TUBITAK) under grant 103E028.
} 
There may be different solutions to the above problem. One may consider balancing the number of messages per processor both in terms of sends and receives. This strategy would then has to partition the computations with the objectives of achieving computational load balance, minimizing total volume of messages, minimizing total number of messages, and also balancing the number of messages sent/received on the per processor basis. However, combining these objectives into a single function to be minimized would challenge the current state of the art. For this reason, we take these problems apart from each other and decompose the overall problem into stages, each of which involving a certain objective. We first use standard models to minimize the total volume of messages and maintain the computational load balance among processors using effective methods, such as graph and hypergraph partitioning. Then, we minimize the total number of messages and maintain a loose balance on the communication volume loads of processors, and in the meantime we address the minimization of the maximum number of messages sent by a single processor. After this stage, the communication pattern is determined. In this paper, we suggest to append one more stage in which the send operations of processors are ordered to address the minimization of the total completion time.

\section{Message Ordering Problem and a Solution}

We make the following assumptions. The computational load imbalance is negligible. All processors begin the pre-communication phase at the same time because of the possible global synchronization points and balanced computations that exist in the other parts of the parallel program. The parallel system has a high latency overhead so that the message transfer time is dominated by the start-up cost due to small message volumes. By the same reasoning, the receive operation is assumed to incur negligible cost to the receiving processor. For the sake of simplicity, the send operations are assumed to take unit time. Under these assumptions, once a send is initiated by a processor at time $t_{i}$, the sending processor can continue with some other operation at time $t_{i+1}$, and the receiving processor receives the message at time $t_{i+1}$. This assumption extends to concurrent messages destined for the same processor. The rationale behind these assumptions is that, the start-up costs for all messages destined for a certain processor truly overlap with each other.

Let send-lists $S_{1}(p)$ and $S_{2}(p)$ denote the set of messages, distinguished by the ranks of the receiving processors, to be sent by processor $P_{p}$ in pre- and postcommunication phases, respectively. For example, $\ell \in S_{1}(p)$ denotes the fact that processor $P_{\ell}$ will receive a message from $P_{p}$ in the pre-communication phase. For $\ell \in S_{1}(p)$, we use $s_{1}(p, \ell)$ to denote the completion time of the message from $P_{p}$ to $P_{\ell}$, i.e., $P_{p}$ issued the send at time $s_{1}(p, \ell)-1$, and $P_{\ell}$ received the message at time $s_{1}(p, \ell)$. We use $s_{2}(p, \ell)$ for the same purpose for the post-communication phase. Let $W$ be the amount of computation performed by each processor. Let

$$
r_{1}(p)=\max _{j: p \in S_{1}(j)}\left\{s_{1}(j, p)\right\}
$$


denote the point in time at which processor $P_{p}$ receives its latest message in the pre-communication phase. Then, $P_{p}$ will enter the computation phase at time

$$
c_{1}(p)=\max \left\{\left|S_{1}(p)\right|, r_{1}(p)\right\},
$$

i.e, after sending all of its messages and receiving all messages destined for it in the pre-communication phase. Let

$$
r_{2}(p)=\max _{j: p \in S_{2}(j)}\left\{s_{2}(j, p)\right\}
$$

denote the point in time at which processor $P_{p}$ receives its latest message in the post-communication phase. Then, processor $P_{p}$ will reach completion at time

$$
c_{p}=\max \left\{c_{1}(p)+W+\left|S_{2}(p)\right|, r_{2}(p)\right\},
$$

i.e., after completing its computational task as well as all send operations in the post-communication phase and after receiving all post-communication messages destined for it. Using the above notation, our objective is

$$
\operatorname{minimize}\left\{\max _{p}\left\{c_{p}\right\}\right\}
$$

i.e, to minimize the maximum completion time. The maximum completion time induced by a message order is called the bottleneck value, and the processor that defines it is called the bottleneck processor. Note that the objective function depends on the time points at which the messages are delivered.

In order to clarify the notations and assumptions, consider a six-processor system as shown in Fig. 1(a). In the figure, the processors are synchronized at time $t_{0}$. The computational load of each processor is of length five-units and shown as a gray rectangle. The send operation from processor $P_{k}$ to $P_{\ell}$ is labeled with $s_{k \ell}$ on the right-hand side of the time-line for processor $P_{k}$. The corresponding receive operation is shown on the left-hand side of the timeline for processor $P_{\ell}$. For example, processor $P_{1}$ issues a send to $P_{3}$ at time $t_{0}$ and completes the send at time $t_{1}$ which also denotes the delivery time to $P_{3}$. Also note that $P_{3}$ receives a message from $P_{5}$ at the same time. In the figure, $r_{1}(1)=c_{1}(1)=t_{5}, r_{2}(1)=t_{10}$ and $c_{1}=t_{15}$. The bottleneck processor is $P_{1}$ with the bottleneck value $t_{b}=t_{15}$.

Reconsider the same system where the messages are sent according to the order as shown in Fig. 1(b). In this setting, $P_{1}$ is also a bottleneck processor with value $t_{b}=t_{11}$.

Note that if a processor $P_{p}$ never stays idle then it will reach completion at time $\left|S_{1}(p)\right|+W+\left|S_{2}(p)\right|$. The optimum bottleneck value cannot be less than the maximum of these values. Therefore, the order given in Fig. 1(b) is the best possible. Let $P_{q}$ and $P_{r}$ be the maximally loaded processors in the pre- and postcommunication phases respectively, i.e., $\left|S_{1}(q)\right| \geq\left|S_{1}(p)\right|$ and $\left|S_{2}(r)\right| \geq\left|S_{2}(p)\right|$ for all $p$. Then, the bottleneck value cannot be larger than $\left|S_{1}(q)\right|+W+\left|S_{2}(r)\right|$. The setting in Fig. 1(a) attains this worst possible bottleneck value. 


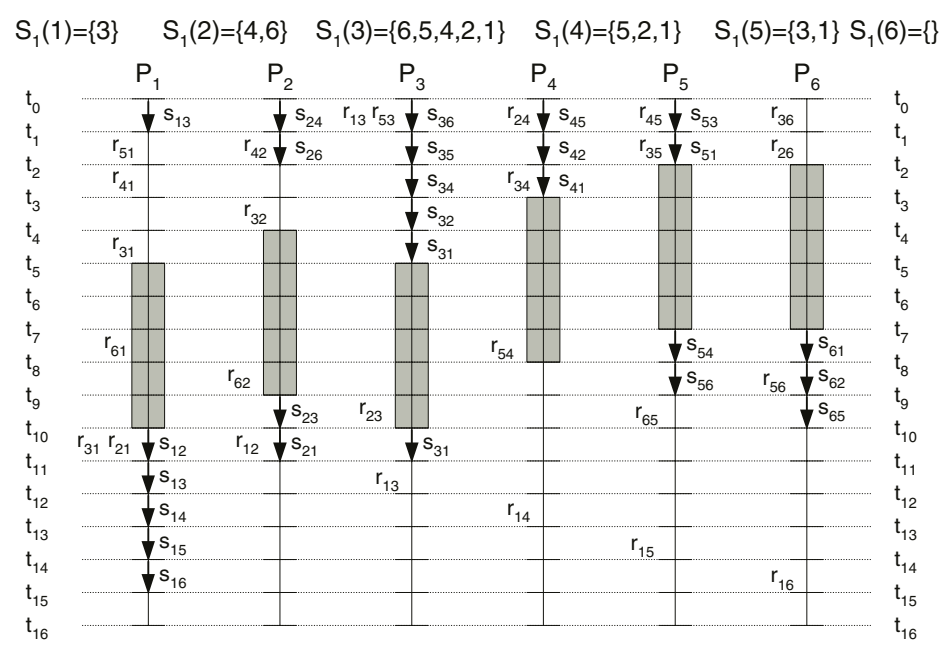

(a) A sample message order which produces worst completion time

$$
S_{1}(1)=\{3\} \quad S_{1}(2)=\{6,4\} \quad S_{1}(3)=\{1,6,2,5,4\} \quad S_{1}(4)=\{1,2,5\} \quad S_{1}(5)=\{1,3\} S_{1}(6)=\{\}
$$

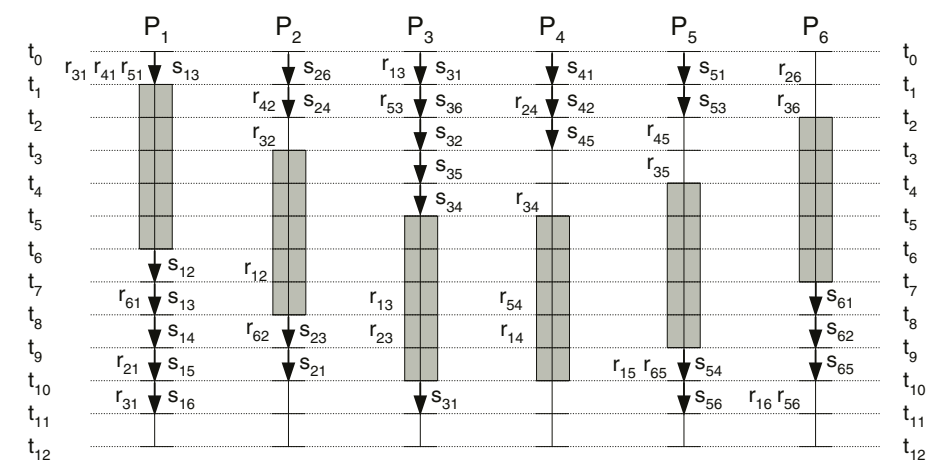

(b) A sample message order which produces best completion time

Fig. 1. Worst and best order of the messages.

Observe that in a given message order, the bottleneck occurs at a processor with an outgoing message. Meaning that, for any bottleneck processor that receives a message at time $t_{b}$, there is a processor which finishes a send operation at time $t_{b}$. Therefore, for a processor $P_{p}$ to be a bottleneck processor we require

$$
c_{p}^{\prime}=c_{1}(p)+W+\left|S_{2}(p)\right|
$$

as a bottleneck value. Hence, our objective reduces to

$$
\operatorname{minimize}\left\{\max _{p}\left\{c_{p}^{\prime}\right\}\right\} \text {. }
$$

Also observe that the bottleneck processor and value remains as is, for any order of the post-communication messages. Therefore, our problem reduces to 
ordering the messages in the pre-communication phase. From these observations we reach the intuitive idea of assigning the maximally loaded processor in the post-communication phase to the first position in each pre-communication sendlist. This will make the processor with maximum $\left|S_{2}(\cdot)\right|$ enter the computation phase as soon as possible. Extending this to the remaining processors we develop the following algorithm. First, each processor $P_{p}$ determines its key-value $\operatorname{key}(p)=\left|S_{2}(p)\right|$. Second, each processor obtains the key-values of all other processors with an all-to-all communication on the key-values. Third, each processor $P_{p}$ sorts its send-list $S_{1}(p)$ in descending order of the key-values of the receiving processors. These sorted send-lists determine the message order in the pre-communication phase, where the order in the post-communication phase is arbitrary.

Theorem 1. The above algorithm obtains the optimal solution that minimizes the maximum completion time.

Proof. We take an optimal solution and then modify it to have each send-list sorted in descending order of key-values.

Consider an optimal solution. Let processor $P_{b}$ be the bottleneck processor finishing its sends at time $t_{b}$. For each send-list in the pre-communication phase, we perform the following operations.

For any $P_{\ell}$ with key $\leq k e y_{\ell}$ where $P_{b}$ and $P_{\ell}$ are in the same send-list $S_{1}(p)$, if $s_{1}(p, \ell) \leq s_{1}(p, b)$, then we are done, if not swap $s_{1}(p, \ell)$ and $s_{1}(p, b)$. Let $t_{s}=s_{1}(p, \ell)$ before the swap operation. Then, we have $t_{s}+W+k e y_{\ell} \leq t_{b}$ before the swap. After the swap we will have $t_{s}+W+k e y_{b}$ and $t_{h}+W+k e y_{\ell}$ for some $t_{h}<t_{s}$, for processors $P_{b}$ and $P_{\ell}$. These two values are less than $t_{b}$.

For any $P_{j}$ with $k e y_{j} \leq k e y_{b}$ where $P_{j}$ and $P_{b}$ are in the same send-list $S_{1}(q)$, if $s_{1}(q, b) \leq s_{1}(q, j)$, then we are done, if not swap $s_{1}(q, b)$ and $s_{1}(q, j)$. Let $t_{s}=s_{1}(q, b)$ before the swap operation. Then, we have $t_{s}+W+k e y_{b} \leq t_{b}$. After the swap operation we will have $t_{s}+W+k e y_{j}$ and $t_{h}+W+k e y_{b}$ for some $t_{h}<t_{s}$ for processors $P_{j}$ and $P_{b}$, respectively. Clearly, these two values are less than or equal to $t_{b}$.

For any $P_{u}$ and $P_{v}$ that are different from $P_{b}$ with $k e y_{u} \leq k e y_{v}$ in a send-list $S_{1}(r)$, if $s_{1}(r, v) \leq s_{1}(r, u)$, then we are done, if not swap $s_{1}(r, u)$ and $s_{1}(r, v)$. Let $t_{s}=s_{1}(r, v)$ before the swap operation. Then, we have $t_{s}+W+k e y_{v} \leq t_{b}$. After the swap operation we will have $t_{s}+W+k e y_{u}$ and $t_{h}+W+k e y_{v}$ for some $t_{h}<t_{s}$, for $P_{u}$ and $P_{v}$ respectively. These two values are less than or equal to $t_{b}$. Therefore, for each optimal solution we have an equivalent solution in which all send-lists in the pre-communication phase are sorted in decreasing order of the key values. Since the sorted order is unique with respect to the key values, the above algorithm is correct.

\section{Experiments}

In order to see whether the findings in this work help in practice we have implemented a simple parallel program which is shown in Fig 2. In this figure, each processor first posts its non-blocking receives and then sends its messages 


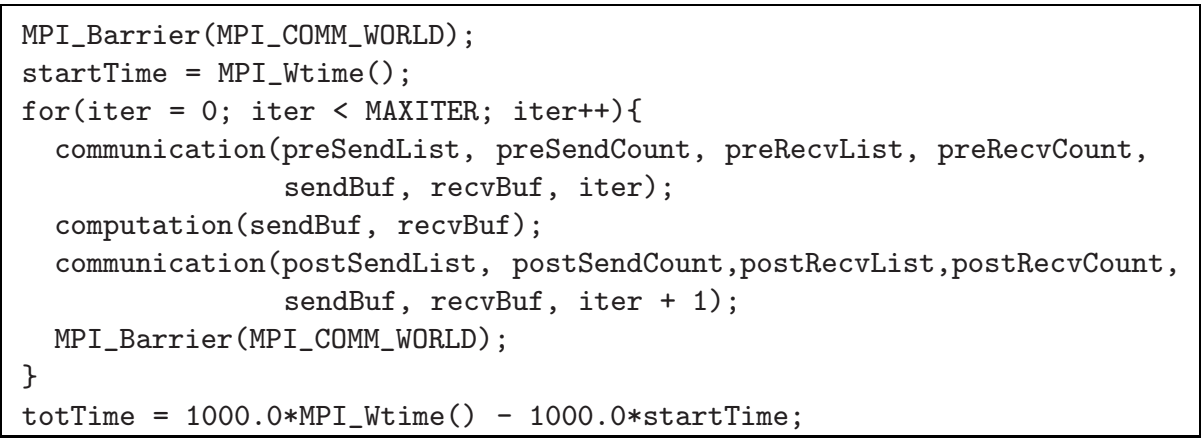

(a) Parallel program segment

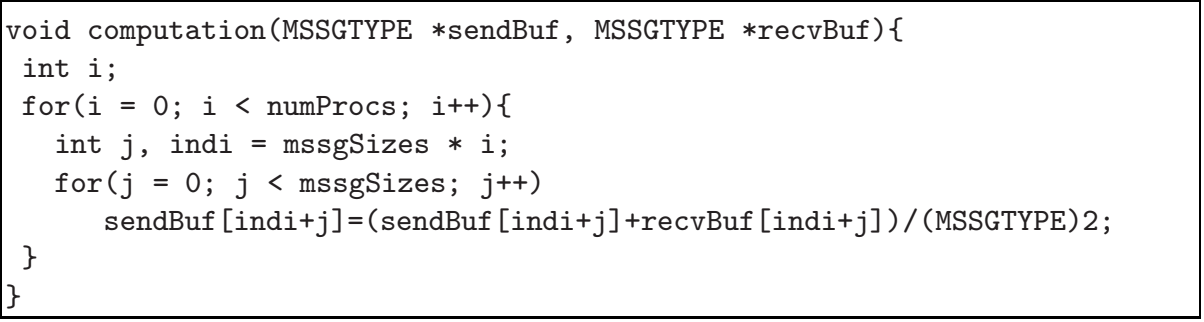

(b) Local computation performed at each processor

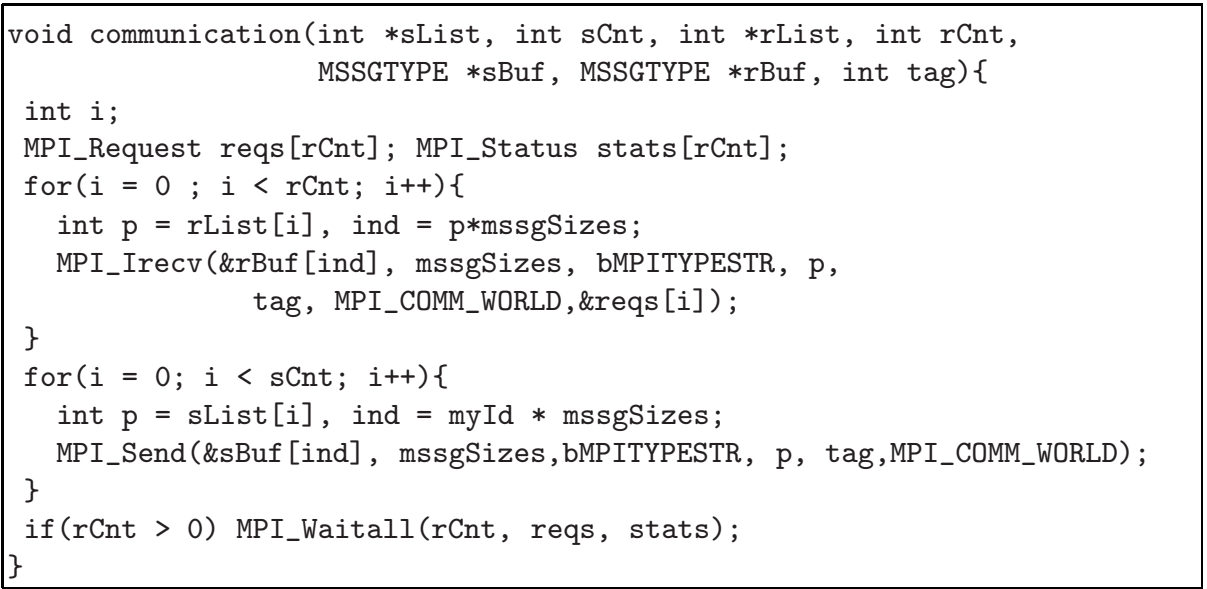

(c) Implementation of pre- and post-communication phases

Fig. 2. A simple parallel program.

in the order as they appear in the send-lists. In order to simplify the effects of the message volume on the message transfer time, we set the same volume for each message. We have used LAM [1] implementation of MPI and mpirun command without -lamd option. The parallel program were run on a Beowulf class [9] PC cluster with 24 nodes. Each node has a 400MHz Pentium-II proces- 
Table 1. Communication patterns and parallel running times on 24 processors.

\begin{tabular}{|c|c|c|c|c|c|c|c|c|c|}
\hline \multirow{4}{*}{ Data } & \multirow{3}{*}{\multicolumn{3}{|c|}{$\begin{array}{c}\text { Communication } \\
\text { pattern }\end{array}$}} & \multirow{4}{*}{$\left|\begin{array}{l}\text { Mssg } \\
\text { order }\end{array}\right|$} & \multicolumn{5}{|c|}{ Completion time } \\
\hline & & & & & \multirow{3}{*}{\begin{tabular}{|l} 
unit \\
Max \\
$\left\{c_{p}^{\prime}\right\}$
\end{tabular}} & \multicolumn{4}{|c|}{ milliseconds } \\
\hline & & & & & & \multicolumn{4}{|c|}{ Message length (bytes) } \\
\hline & $\min$ & $\operatorname{lax}$ & tot & & & 8 & 64 & 512 & 1024 \\
\hline 1-PRE & 5 & 21 & 290 & best & 38 & 4.3 & 4.4 & 5.5 & 7.2 \\
\hline 1-POST & 6 & 22 & 358 & worst & $\overline{42}$ & 4.8 & 5.0 & 6.2 & 7.8 \\
\hline 2-PRE & 3 & 23 & 313 & best & 39 & 4.9 & 5.0 & 6.0 & 7.3 \\
\hline 2-POST & 11 & 22 & 370 & worst & 45 & 5.3 & 5.4 & 6.7 & 7.8 \\
\hline 3-PRE & 10 & 23 & 490 & best & 45 & 6.3 & 6.4 & 7.8 & 9.7 \\
\hline 3-POST & 15 & 23 & 504 & worst & 46 & 6.6 & 6.6 & 8.2 & 10.1 \\
\hline 4-PRE & 6 & 22 & 312 & best & 41 & 4.5 & 4.6 & 5.9 & 7.3 \\
\hline 4-POST & 10 & 20 & 356 & worst & $\overline{42}$ & 5.3 & 5.6 & 6.8 & 8.2 \\
\hline 5-PRE & 5 & 23 & 228 & best & 36 & 4.0 & 4.1 & 4.9 & 5.9 \\
\hline 5 -POST & 7 & 13 & 228 & worst & 36 & 4.4 & 4.6 & 5.6 & 6.6 \\
\hline 6-PRE & 1 & 23 & 212 & best & 35 & 4.1 & 4.1 & 5.1 & 6.0 \\
\hline 6-POST & 4 & 17 & 236 & worst & 40 & 4.5 & 4.6 & 5.8 & 6.7 \\
\hline 7-PRE & 3 & 20 & 226 & best & 29 & 3.7 & 3.7 & 4.5 & 5.3 \\
\hline 7-POST & 7 & 17 & 253 & worst & 37 & 3.9 & 3.9 & 5.0 & 5.9 \\
\hline 8-PRE & 2 & 23 & 267 & best & 43 & 4.7 & 4.7 & 6.1 & 7.6 \\
\hline 8-POST & 4 & 22 & 278 & worst & 45 & 5.7 & 5.9 & 7.0 & 8.1 \\
\hline 9-PRE & 3 & 16 & 167 & best & 35 & 3.7 & 4.0 & 4.8 & 5.6 \\
\hline 9-POST & 4 & 20 & 273 & worst & 36 & 4.3 & 4.3 & 5.3 & 6.0 \\
\hline 10-PRE & 2 & 23 & 300 & best & 46 & 4.7 & 4.7 & 6.3 & 8.0 \\
\hline 10-POST & 10 & 23 & 316 & worst & 46 & 5.6 & 5.7 & 7.1 & 8.3 \\
\hline & $\bar{V}$ & & put & tion & & 0.00 & 0.01 & 0.06 & 0.11 \\
\hline
\end{tabular}

sor and $128 \mathrm{MB}$ memory. The interconnection network is comprised of a $3 \mathrm{COM}$ SuperStack II 3900 managed switch connected to Intel Ethernet Pro 100 Fast Ethernet network interface cards at each node. The system runs Linux kernel 2.4.14 and Debian GNU/Linux 3.0 distribution.

We extracted the communication patterns of some row-column-parallel sparse matrix-vector multiply operations on 24 processors. Table 1 lists minimum and maximum number of send operations per processor under columns min and max. Total number of messages is given under the column tot.

For each test case, we have run the parallel program of Fig. 2 with small message lengths of $8,64,512$, and 1024-bytes to justify the practicality of the assumptions made in this work. We have experimented with the best and worst orders. The best message orders are generated according to the algorithm proposed in $\S 2$. The worst message orders are obtained by sorting the pre-communication send-lists in increasing order of the key-values of the receiving processors. In all cases, we used the same message order in the post-communication phase. The running are presented in milliseconds in Table 1 . We give the best among 20 runs (see [6] for choosing best in order to obtain reproducible results). In the table, we also give $\max _{p}\left\{c_{p}^{\prime}\right\}$ for worst and best orders with $W=0$. In all cases, the best order always gives better completion time than the worst order. In theory, however, we did not expect improvements for the 5th and 10th cases, in which 
the two orders give the same bottleneck value. This unexpected outcome may be resulting from the internals of the process that handles the communication requests. We are going to investigate this issue.

\section{Conclusion}

In this work, we addressed the problem of minimizing maximum completion time of a certain class of parallel program segments in which there is a smallto-medium grain computation between two communication phases. We showed that the order in which the messages are sent affects the completion time and showed how to order the messages optimally in theory. Experimental results on a PC cluster verified the existence of the specified problem and the validity of the proposed solution. As a future work, we are trying to set up experiments to observe the findings of this work in parallel sparse matrix-vector multiplies. A generalization of the given problem addresses parallel programs that have multiple computation phases interleaved with communications. This problem is in our research plans.

\section{References}

1. G. Burns, R. Daoud, and J. Vaigl. LAM: an open cluster environment for MPI. In John W. Ross, editor, Proceedings of Supercomputing Symposium '94, pages 379-386. University of Toronto, 1994.

2. Ü. V. Çatalyürek and C. Aykanat. Hypergraph-partitioning based decomposition for parallel sparse-matrix vector multiplication. IEEE Transactions on Parallel and Distributed Systems, 10(7):673-693, 1999.

3. Ü. V. Çatalyürek and C. Aykanat. A fine-grain hypergraph model for $2 \mathrm{~d}$ decomposition of sparse matrices. In Proceedings of International Parallel and Distributed Processing Symposium (IPDPS), April 2001.

4. Ü. V. Çatalyürek and C. Aykanat. A hypergraph-partitioning approach for coarsegrain decomposition. In Proceedings of Scientific Computing 2001 (SC2001), pages 10-16, Denver, Colorado, November 2001.

5. J. J. Dongarra and T. H. Dunigan. Message-passing performance of various computers. Concurrency-Practice and Experience, 9(10):915-926, 1997.

6. W. Gropp and E. Lusk. Reproducible measurements of mpi performance characteristics. Tech. Rept. ANL/MCS-P755-0699, Argonne National Lab., June 1999.

7. B. Hendrickson and T. G. Kolda. Graph partitioning models for parallel computing. Parallel Computing, 26:1519-1534, 2000.

8. B. Hendrickson and T. G. Kolda. Partitioning rectangular and structurally unsymmetric sparse matrices for parallel processing. SIAM J. Sci. Comput., 21(6):20482072, 2000.

9. T. Sterling, D. Savarese, D. J. Becker, J. E. Dorband, U. A. Ranaweke, and C. V. Packer. BEOWULF: A parallel workstation for scientific computation. In Proceedings of the 24th International Conference on Parallel Processing, 1995.

10. B. Uçar and C. Aykanat. Minimizing communication cost in fine-grain partitioning of sparse matrices. In A. Yazıcı and C. Şener, editors, in Proc. ISCISXVIII-18th Int. Symp. on Computer and Information Sciences, Antalya, Turkey, Nov. 2003.

11. B. Uçar and C. Aykanat. Encapsulating multiple communication-cost metrics in partitioning sparse rectangular matrices for parallel matrix-vector multiplies. SIAM J. Sci. Comput., 25(6):1837-1859, 2004. 